\title{
Work-Life Integration and Time Management Strategies
}

\author{
Lindsey Gade, MD ${ }^{1}$ Heather L. Yeo, MHS, MD, MBA, MS, FACS, FASCRS 2,3 \\ ${ }^{1}$ Department of Surgery, NewYork-Presbyterian Hospital/Weill \\ Cornell Medicine, New York, New York \\ 2 Department of Surgery, NewYork-Presbyterian Queens, Queens, New York \\ ${ }^{3}$ Department of Healthcare Policy and Research, Weill Cornell \\ Medicine, New York, New York \\ Address for correspondence Heather L. Yeo, MHS, MD, MBA, MS, \\ FACS, FASCRS, Department of Surgery, NewYork-Presbyterian \\ Hospital/Weill Cornell Medicine, 525 East 68th St., New York, NY \\ 10065 (e-mail: hey9002@med.cornell.edu).
}

Clin Colon Rectal Surg 2019;32:442-449.

Abstract

Keywords
- work-life integration
- short-/long-term
goals
- schedule
expectations
- environment

When choosing a career as a surgeon, we knowingly dedicate ourselves to a lifetime of service and education. Our commitment as physicians is but one of many commitments in the larger scheme of life where we function as family members, friends, athletes, and numerous other roles. Work and life are often described as two separate entities diametrically opposed to each other. In reality, personal and professional goals are part of a continuum where work is a major part of our lives and who we are as people and is not necessarily separate from the others. The goal-directed nature with which we approach our responsibilities as surgeons should be applied to all domains of life. As we progress along the training paradigm from intern to attending, control over time allocation increases. Understanding oneself, determining priorities, applying realistic expectations, cultivating a supportive environment, setting personal and professional goals, and being held accountable for progress and completion of these goals will allow us to utilize limited time efficiently to achieve what we individually desire from life.
Work-life integration means coordinating and blending elements of life into a unified whole where work, family, friends, and self are valued, and time is allocated proportionally. Traditionally, surgeons have struggled to fulfill the demands of each sphere with the expectation that they should lead a work-dominated life. The consequences of this mindset are a much higher rate of burnout than the general population (53\% vs $28 \%$ ), failed relationships, physical and mental illness, poor work performance, and/or career change. ${ }^{1-5}$ As a result, we must move away from this traditional mindset and reformulate what it means to be a surgeon using an array of tips and tools to help us successfully integrate all spheres of life.

To understand how to improve, we must first understand how this culture evolved. The term "resident" derives its origin from the fact that early physicians in training spent the majority of their time residing in the hospital. The American College of Surgeons was formed in 1913, the American Board of Surgery was formed in 1937, and the Conference Committee on Graduate Training in Surgery was formed in 1950 (later the
Residency Review Committee for Surgery), yet no governing body instituted rules or regulations regarding resident duty hours, supervision, or work environment. Residents were afforded an unfettered operative and clinical experience at the high cost of personal health and productivity. A multitude of observational studies documented the detrimental effects of unrestricted work hours and sleep deprivation on residents, including increased somnolence while performing procedures, ${ }^{6}$ increased motor vehicle accidents, ${ }^{7-11}$ negative effect on mood, affect, and cognition, ${ }^{12-17}$ depression, ${ }^{14,18-21}$ and obstetric complications in pregnant female residents. ${ }^{22-25}$ Despite the negative impact, residents continued to work unrestricted hours until a major error committed by an overworked, unsupervised first-year resident resulted in a young woman's death.

The acceptance and enforcement of regulated resident work hours has significant implications for the culture of medicine and surgery as a whole. Young surgeons expect to achieve satisfaction in all spheres of their life and no longer feel
Issue Theme Surgeon Health; Guest Editor: Jennifer S. Davids, MD, FACS, FASCRS
Copyright $\odot 2019$ by Thieme Medical Publishers, Inc., 333 Seventh Avenue, New York, NY 10001, USA. Tel: +1(212) 584-4662.
DOI https://doi.org/ 10.1055/s-0039-1693011. ISSN 1531-0043. 
that a career in surgery means sacrificing life outside of the hospital. ${ }^{26}$ This is evidenced by the AAMC's report that in 2011, physicians younger than 35 years worked 3.5 to 5.4 hours less per week as compared with physicians in $1980 .{ }^{27}$ Millennial surgeons may desire to "have it all," but to be successful in all they endeavor, they will need to utilize tools of organization and efficiency, as described in the following.

There is a large body of literature on time management and work-life integration, most of its suggesting the importance of goal setting and time management strategies. In this study, we will review some suggestions related to integrating these strategies into the life of busy surgeons and will give some practical strategies and tools for moving this forward.

\section{Tools for a Successful Integration of Personal and Professional Goals}

Time is a limited resource and must be treated as such. There are only 24 hours in a day, and having it all does not mean having everything you want at once. Priorities must be chosen, goals must be established, and a timeline must be assigned. The following tools are necessary for creating an individualized, realistic plan to accomplish your goals.

\section{Know yourself, keep realistic expectations, and make a schedule}

Aristotle said, "Knowing yourself is the beginning of all wisdom." To successfully integrate personal and professional goals, it is necessary to understand yourself, define your priorities, and set realistic goals rooted in these priorities. If you have not thought about what you need, take a moment to reflect on the last month and catalog your activities. What are the activities you engage in most frequently? What activities are fulfilling but seldom? Be sure to consider time spent on research, reading, mentoring, exercise, and family responsibilities (i.e., kid's homework, sports). Make a list that includes all of these activities. From this list, choose the most important activities and make a new list. Document the associated time commitment next to each activity. -Fig. 1 demonstrates a potential list of activities with their associated weighted importance and the corresponding action that should be taken according to the importance and urgency of the task.

Making a schedule and adhering to it is essential to maximizing productivity. Make a weekly schedule with the appropriate amount of time dedicated to the necessary activities. When making your schedule, do not overcommit yourself or include activities outside of those deemed necessary. Psychology studies of consumers and employees have demonstrated that unmet expectations of any sort are associated with dissatisfaction, emotional exhaustion, and decreased organizational commitment. ${ }^{28-30}$ Making a schedule that you cannot legitimately fulfill sets you up for failure and limits productivity. - Fig. 2 provides a list of time and task management applications that can be used to help achieve maximum productivity and to stay on track when working toward your goals. It is important to manage not only your own expectations but also those of close family and friends. While it is often difficult to plan months ahead in this specialty, it is necessary to reach out to those closest to you to find out about important, inflexible activities you may be expected to take part in. Make schedule requests as early as possible and keep your loved ones informed so that they may plan appropriately. Sharing your personal daily calendar with loved ones is an easy way to keep those close to you informed of your availability.

\begin{tabular}{|c|c|c|}
\hline & Important & Less Important \\
\hline Urgent & $\begin{array}{c}\text { Action: Do Now } \\
\text { Crashing Patient } \\
\text { Terminally Ill Family Member } \\
\text { Sick Child } \\
\text { Medical Emergencies } \\
\text { Deadline for Presentation at Major } \\
\text { Conference } \\
\text { Grant Deadline }\end{array}$ & $\begin{array}{c}\text { Action: Delegate/Push Back } \\
\text { Household Chores } \\
\text { Project Deadlines } \\
\text { Paying Bills } \\
\text { Some Emails/Calls/Meetings } \\
\text { Favors for Others } \\
\text { Attending Rounds } \\
\text { Returning Patient Phone Calls }\end{array}$ \\
\hline $\begin{array}{l}\text { Less } \\
\text { Urgent }\end{array}$ & $\begin{array}{c}\text { Action: Plan/Do Soon } \\
\text { Research Projects } \\
\text { Self Care/Exercise } \\
\text { Family Events } \\
\text { Attending Academic Conferences } \\
\text { Attending Committee Meetings } \\
\text { Mentoring } \\
\text { Skill Workshops/Practice } \\
\text { Book Chapter for Senior Faculty } \\
\text { Financial Planning } \\
\text { Recreation } \\
\text { Relationship Building/Networking }\end{array}$ & $\begin{array}{c}\text { Action: Avoid/Do Last } \\
\text { Some Phone Calls/Meetings } \\
\text { Busy Work } \\
\text { Trivia } \\
\text { TV Watching } \\
\text { Home Improvement Projects } \\
\text { Giving Ancillary Lectures }\end{array}$ \\
\hline
\end{tabular}

Fig. 1 A simplified time management grid to help organize priorities and direct action based on priority. (Adapted from Stephen Covey, The Seven Habits of Highly Effective People. New York, NY: Free Press; 2004:151.) 


\begin{tabular}{|c|c|c|c|c|c|c|c|c|}
\hline & toggl & asana & scoro & Clarizen & Trello & REPLICON & Wrike & Timecamp \\
\hline $\begin{array}{l}\text { Project + Task } \\
\text { Management }\end{array}$ & $\checkmark$ & $\checkmark$ & $\checkmark$ & $\checkmark$ & $\checkmark$ & $\checkmark$ & $\checkmark$ & $\checkmark$ \\
\hline Time Tracking & $\checkmark$ & & $\checkmark$ & $\checkmark$ & & $\checkmark$ & $\checkmark$ & $\checkmark$ \\
\hline $\begin{array}{l}\text { Resource } \\
\text { Planning }\end{array}$ & $\checkmark$ & & $\checkmark$ & $\checkmark$ & & $\checkmark$ & $\checkmark$ & \\
\hline $\begin{array}{l}\text { Shared } \\
\text { Calendar }\end{array}$ & & $\checkmark$ & $\checkmark$ & $\checkmark$ & $\checkmark$ & & $\checkmark$ & \\
\hline $\begin{array}{l}\text { Real - Time Key } \\
\text { Performance } \\
\text { Indicator } \\
\text { Dashboard }\end{array}$ & & & $\checkmark$ & $\checkmark$ & & $\checkmark$ & $\checkmark$ & \\
\hline Work Reporting & $\checkmark$ & $\checkmark$ & $\checkmark$ & $\checkmark$ & & $\checkmark$ & $\checkmark$ & $\checkmark$ \\
\hline Cloud Service & $\checkmark$ & $\checkmark$ & $\checkmark$ & $\checkmark$ & $\checkmark$ & $\checkmark$ & $\checkmark$ & $\checkmark$ \\
\hline
\end{tabular}

Fig. 2 Time management applications with the listed services to help with organization, performance tracking, and goal measurement.

Making a family calendar is a good way to ensure that all family members are accounted for and aware of each other's important events. Assign each family member a different color or unique identifier. Obtain your children's school calendars at the outset of the school year and add vacation days, conferences, and important school events to that calendar. Add inflexible work commitments and conferences as soon as they are determined for both you and your spouse. Plan vacation time around inflexible or important events (i.e., birthdays, reunions, extended family events). - Fig. 3 provides a list of popular calendar applications that are geared toward family organization.

\section{Define short- and long-term professional and personal} goals

Self-evaluation and the establishment of short- and longterm goals are keys to maximizing productivity. The goalsetting theory states that consciously setting a challenging, specific goal will lead to higher performance. ${ }^{31-35}$ Begin by identifying long term personal and professional goals and write them down. Next, choose a "partner," someone close to you personally and/or professionally, with whom you will share your goals. By doing this, you not only solicit a source of support, but you also have identified someone to hold you accountable for your progress. Next, devise a strategy to achieve these goals using a series of short-term goals (subgoals $)^{31,32}$ and keep your partner informed. Again, your goals and timeline must be realistic. If you find that you are failing to complete your goals in the time allotted, "intervention" by your partner may be necessary to determine if you are setting realistic goals and to reformulate your schedule if needed. ${ }^{36}$ Goal setting in itself is a skill that is refined with practice and experience.

For example, consider applying for an R01 grant. You have collected your baseline data and feel ready to address a topic of larger significance with the support of an R01 grant. While

\begin{tabular}{|c|c|c|c|c|c|}
\hline & cozi & hub & FamilyWall & Famjama & SKEDi \\
\hline Color-coded by Family Member & 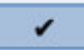 & $\checkmark$ & $\checkmark$ & 2 & 2 \\
\hline Filters for Sorting & $\checkmark$ & & $\checkmark$ & $\checkmark$ & $\checkmark$ \\
\hline $\begin{array}{l}\text { Shared To-do Checklists with } \\
\text { Ability to Assign Tasks (Chores, } \\
\text { Groceries) }\end{array}$ & $\checkmark$ & $\checkmark$ & $\checkmark$ & $\checkmark$ & $\checkmark$ \\
\hline Reminders/ Notifications & $\checkmark$ & & $\checkmark$ & 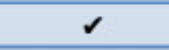 & \\
\hline Shared Notepad/ Journal & $\checkmark$ & $\checkmark$ & $\checkmark$ & $\checkmark$ & \\
\hline $\begin{array}{l}\text { Integrates with Existing } \\
\text { Calendars }\end{array}$ & $\checkmark$ & $\checkmark$ & $\checkmark$ & & $\checkmark$ \\
\hline Shared Social Media & & $\checkmark$ & $\checkmark$ & & \\
\hline $\begin{array}{l}\text { Real-Time Location Tracking } \\
\text { and Check-In }\end{array}$ & & & $\checkmark$ & & \\
\hline Emergency Alert Button & & & $\checkmark$ & & \\
\hline In-App Messaging & & & $\checkmark$ & $\checkmark$ & \\
\hline Web App Only & & & & $s$ & \\
\hline
\end{tabular}

Fig. 3 Calendar applications that integrate all family members' activities and provide the listed services to assist in family organization and time management. 
the amount of time allotted for this goal depends on the veracity of your background research, let us estimate that this will take roughly a year to complete. Divide this end goal into tangible monthly goals using a monthly schedule that you and your mentor will follow to ensure you stay on track. These monthly goals may include familiarizing yourself with grant criteria, obtaining a mentor experienced in your field, writing grant aims, assembling preliminary data, enlisting senior faculty or expert consultants for input, and writing grant drafts that will cycle through many edits before submission.

Now consider running a marathon. As a means of restoring fitness and as a personal challenge, you have made competing in a marathon a priority and now must enact a plan. Again, the amount of time needed for preparation depends upon your current fitness, but let us estimate that it will take roughly 6 months to appropriately prepare. Make a schedule of monthly goals that may include finding a partner to train with, subscribing to organizations that sponsor shorter races (i.e., New York Road Runners), blocking out time to run twice a week, blocking out time to run three times a week, competing in a 5-mile race, and competing in a half marathon.

\section{Create a supportive environment conducive to success}

Prior to beginning surgery, we ensure that the necessary instruments and implants are in the room, position the patient, display necessary imaging, and call a timeout to ensure that the proper precautions have been taken and that everyone is in agreement regarding the procedure that will be performed. Just as a good setup will make a procedure run smoothly, constructing a supportive environment is necessary to achieve personal and professional success. Defining your environment, or the surroundings or conditions in which a person lives or operates, involves making decisions about not only where you will be but perhaps equally important who will be there. While some of these factors may be out of your control, making informed decisions regarding aspects you can control will make your life daily life easier and ultimately more fulfilling.

Determining long-term goals is necessary to inform decisions regarding your environment. While it is difficult to appreciate what your needs will be 10 years from now, understanding career options and evaluating yourself within the context of these options early on will facilitate decisionmaking, goal formation, and time allocation. Factors to be considered include the following:

- Practice type: the type of practice, private or academic, must also be considered early on. Private practice, or a business that is not controlled or paid for by the government or a larger company such as a hospital, may be as small as a single physician, but most commonly it occurs as group practices with several surgeons. ${ }^{37}$ As there are no medical students or residents, emphasis is mostly on clinical practice, but motivated surgeons may pursue clinical research as well. Academic medicine includes both academic medical centers and institutions that are both a medical school and a hospital, as well as affiliated community hospitals. Academic surgeons are members of a medical school department of surgery and serve as educators, clinicians, and researchers. Academically affiliated surgeons represent a mix between private practice surgeons and academic surgeons as they serve as educators and clinicians but are not required to participate in research. In a survey of 1,215 private practice surgeons and 317 academic surgeons, significant differences were found in call, vacation, benefits, retirement, and salary with private practice surgeons having more call, vacation time, fewer benefits, earlier retirement, and a higher income than academic surgeons. ${ }^{37}$ Practice content also differed between the two with private practice surgeons performing more elective cases, cholecystectomies, appendectomies, and femoropopliteal bypasses, whereas academic surgeons performed more emergent cases, breast surgeries, and colectomies. Private practice surgeons saw more patients and performed more procedures each week than academic surgeons who devoted more time to administrative business, teaching, and research activities. There was no significant difference in total hours of work between private and academic surgeons. Both private and academic surgeons reported high career satisfaction. Regardless of your choice to pursue private or academic practice, it is necessary to find a practice whose vision is in-line with your own and who will provide the necessary resources to achieve your goals.

- Academic considerations: in academic practice, resources to be considered include an appropriate amount of dedicated research time, funding for equipment and/or support staff, and mentorship in your specialty. It is also necessary to understand the requirements for promotion as the time and commitment required to achieve promotion may not suit your goals.

- Private practice considerations: in private practice, the size and structure of the practice has implications for your lifestyle, and decisions must be made in accordance with your personal and professional goals. If your goal is ultimately to become a partner in owning a practice, this often means taking more call and working longer hours upfront with the understanding of increased monetary compensation in the future. This will also mean increased administrative work in managing insurance and compliance, and maintaining a referral base. If your personal goals take priority, a salaried position at a large practice may be more desirable. Working in a medium-to-large practice where compensation may be salary based and/or incentivized by productivity or quality measures represents another option that offers flexibility in time allocation and the opportunity for increased monetary compensation. For each of these scenarios, it is important to remember that networking and maintaining a referral base are part of the job and require dedicated time allotment. 
- Work environment: regardless of which practice type you choose, a strong mentor is invaluable as this will be the person who helps define your goals, holds you accountable for achieving these goals, and who will support you clinically. A National Institutes of Health study of more than 1,700 clinicians receiving mentoring for career development demonstrated that increased time spent with one's mentor and collegiality of mentoring significantly increased job satisfaction. ${ }^{38}$ The culture of a practice may also impact your productivity and overall satisfaction. A survey of more than 4,000 general surgery residents demonstrated that work and life satisfaction was three times higher for residents who maintained collegial relationships with attendings. ${ }^{39}$ Taking time to gauge the involvement of faculty/ coworkers with each other and their involvement with departmental/practice initiatives will help determine the supportive or malignant climate of the environment.

- Geographic location: when choosing a location to practice, programmatic factors should contribute to your choice, but personal factors should be valued with equal importance. If partnered, your significant other's needs and priorities must be established especially with consideration for work opportunities, distance from family, or even city versus suburban life. It is important to choose a location where you will have a strong support system, which often means living close to family and friends. In a survey of women surgeons, $88 \%$ identified social support networks as a key strategy for success in their career. ${ }^{40}$ These are often the partners you will choose to help accomplish personal goals and are especially valuable with regard to childcare. While there are many benefits of having friends and family in close proximity, time distribution may be more difficult and schedule adherence becomes more important. Specific individual logistical considerations, such as commute, parking, and proximity to schools and/or childcare, should also be considered to maximize time efficiency.

- Family: male surgeons are more likely than female surgeons to be married and to have children. ${ }^{5,41}$ Female surgeons are more likely to postpone childbirth until after residency and are less likely to have their spouse acting as the primary caregiver as compared with male surgeons. ${ }^{5,41,42}$ Female surgeons report more frequent career conflicts with their spouse and are more likely to have experienced a recent argument with their spouse regarding work-home responsibilities. ${ }^{5}$ Taken together, this information indicates that there is differential stress placed on female surgeons who are parents as compared with males and that this is likely due to the stresses associated with their role as the primary caregiver. Choosing a partner is one of the biggest decisions that any human makes, but in this profession, it is particularly important to choose a supportive partner who understands the demands of a surgical career and who will support your personal and professional goals.

According to the Bureau of Labor Statistics, $47.5 \%$ of American couples are dual-career couples. ${ }^{43}$ Dual-career couples may face added challenges including managing travel schedules, living in different cities, coordinating childcare, tending to household duties, and/or simply spending time together. The following actions can help foster and maintain a healthy relationship in the face of demanding careers:

- Make a long-term plan: define your 5- to 10-year plan individually and share this plan with your partner. It is possible for both partners to accomplish their goals only when goals are shared, understood, and valued. A realistic and detailed plan to accomplish these goals can then be devised. This may involve trade-offs and periods of sacrifice, but this should be balanced in such a way that maintains equality in the relationship and sustains a supportive environment. ${ }^{44,45}$

- Manage expectations: to support each other appropriately, communication is key. Partners must communicate their own needs and understand the needs of their partner. Expectations should be specific and detailed with regard to all aspects of life including, but not limited, to the following: income and budget, household chores, child care, amount of time spent together, travel, daily meals, and even preferred methods of communication. Be direct and clear about your needs and be open to compromise when your needs are not in sync. Once expectations have been established, check in with your partner periodically to assess whether or not expectations are being met. ${ }^{44}$

- Schedule time with your partner: schedule time to spend with your partner as you would for a regular business meeting. Using a shared calendar, block out time to spend together with as much regularity as your schedule will allow. $^{44}$

- Bring your partner and colleagues together: as phone calls, e-mails, and projects often take place at home, you may become familiar with your partner's colleagues without having met them. Use opportunities such as company parties, fundraisers, and family events to spend time with your colleagues and partner together. It not only allows your partner to get to know the people you spend the majority of your day with but also provides an opportunity for your colleagues to understand and become sensitive to your personal priorities. ${ }^{44}$

Childbearing and parental duties add a significant amount of responsibility and stress to any parent's life, but this is felt more intensely by those with significant professional commitments such as surgeons. Having a support system in place can help diffuse some of the responsibility. This may include involving extended family to provide care, enrolling in a daycare facility, and/or hiring a full-time nanny. Seeking a program or practice that has structured policies and programs regarding pregnancy and childcare will be beneficial for all surgeons and trainees who have children or foresee having children in the near future. Stanford has enacted a pilot "time-banking" program, whereby doctors earn credits for often overlooked academic and professional activities including mentoring, 
teaching, and serving on committees, which can be used for work- or home-related services including housecleaning, babysitting, handyman services, dry cleaning, grant writing help, speech training, and elderly care. ${ }^{46}$ While this is a unique program, outsourcing chores is something that we can and should take advantage of. Time spent with family should be focused on activities that enrich our relationships with our loved ones.

\section{Major Life Events}

The majority of surgeons will have spent their twenties and thirties in training and their thirties and forties in developing their career. During this time period, we will experience some of life's most formative events. While pregnancy and marriage represent positive, foreseen events, we inevitably will experience personal tragedies including family members' illness or death, divorce, financial instability, and personal illness. A 2013 cross-sectional study of all residents and fellows at Duke University revealed that $96 \%$ of trainees sustained major life events, and 33\% of trainees suffered the death of a close family member. ${ }^{47}$ While no such study has been performed for practicing surgeons, it stands to reason that as we age, death and illness become more common. Often, we take for granted the health of our loved ones. Time with aging or ill family members is fleeting and must be prioritized. Guilt associated with increasing coworkers work burden may stop us from fulfilling familial obligations. Communicate with your colleagues and do not be afraid to ask for help. While responsibility toward our patients and coworkers is important, adjustments can always be made and the machine will roll on with or without you. There will always be opportunities to make up work, but you will never be able to get time back with a dying loved one. In 10 years, will you regret missing out on valuable time spent with a loved one who has died or will you regret missing work and the associated professional costs? Trainees and surgeons should be supported in taking the necessary time away from work to care for their family. When caring for an elderly or ill parent, you must employ multiple resources including support from colleagues, support/care from extended family, home health services, and occupational/ rehabilitation services. When developing short- and longterm goals, trainees and surgeons must take family needs into account, as remaining in close proximity to family members will allow for a more active role in the care of family members. Again, this priority and the associated time commitment must be appropriately accounted for when defining goals and creating a schedule.

\section{Special Considerations for the Surgical Resident}

While residents have little control over their schedule, the aforementioned strategies can still be applied to allow residents to achieve a higher quality of life during residency. Nonetheless, it is important to understand the barriers to work-life integration that residents with families face, the importance of programmatic support, and the changes that need to be made to better support our trainees with families. Roughly $50 \%$ of surgical residents are married and roughly $25 \%$ have children. ${ }^{48}$ Male residents are more likely to be married and have children than female residents. ${ }^{48}$ The effects of being married and having children are felt positively by male residents who report increased work satisfaction but not by females who report no such increase. Furthermore, program directors felt that becoming a parent negatively affects female trainees' work, decreases their well-being, and increases coworkers' burden of work. ${ }^{49}$ Regardless of sex, having children during residency increases work-induced family strain. ${ }^{48}$ Only $38 \%$ of programs have onsite childcare at their hospitals. Programs still lack national uniformity in policies regarding parental leave and lactation, but larger programs (more than six residents per class) are more likely to have official policies regarding parental leave, lactation facilities, and access to daycare. ${ }^{49}$ While it may be advisable for some to delay starting a family until the conclusion of residency, this is not always an option and residents should feel supported should they choose to start a family during residency. Until policies regarding maternity leave, paternity leave, and breastfeeding become uniform, we need to be aware of our trainees' familial and personal needs and make provisions accordingly. Programs should try to provide as much schedule flexibility as possible within the ACGME (Accreditation Council for Graduate Medical Education) guidelines, make provisions for lactation for new mothers, and assist new parents in planning for and securing daycare assistance as the demands of parenthood in residency are large. We must support the needs of our trainees as they encounter major life events, whether positive or negative.

\section{Conclusion}

Successful integration of personal and professional goals requires self-evaluation, prioritization, and the creation of realistic short- and long-term goals that have a realistic and timely plan of execution. The enlistment of a professional and/or personal "partner" helps ensure accountability and success. Goals are best achieved by choosing an environment that contains resources and people who will provide the necessary support. As surgeons, we assume many roles in life as family members, clinicians, scientists, and friends, and each surgeon will define success differently. Through a process of continuous self-evaluation and evaluation of our surroundings, we can prioritize our goals and maximize what is important to us.

Conflict of Interest

None declared.

Acknowledgments

L. G. received support from the Agency for Healthcare Research and Quality, National Research Service Award (T32-HS000066-23). 


\section{References}

1 Dimou FM, Eckelbarger D, Riall TS. Surgeon burnout: a systematic review. J Am Coll Surg 2016;222(06):1230-1239

2 Fernandez Nievas IF, Thaver D. Work-life balance: a different scale for doctors. Front Pediatr 2015;3(December):115

3 Shanafelt TD, Hasan O, Dyrbye LN, et al. Changes in burnout and satisfaction with work-life balance in physicians and the general US working population between 2011 and 2014. Mayo Clin Proc 2015;90(12):1600-1613

4 Swensen S, Kabcenell A, Shanafelt T. Physician-organization collaboration reduces physician burnout and promotes engagement: the Mayo Clinic experience. J Healthc Manag 2016;61(02):105-127

5 Dyrbye LN1. Shanafelt TD, Balch CM, Satele D, Sloan J, Freischlag J. Relationship between work-home conflicts and burnout among American surgeons: a comparison by sex. Arch Surg 2011;146 (02):211-217

6 Nightline AN. Surgeons in training, running on empty; young doctors spend 110 hours per week or more doing their residencies [transcript]. "Nightline." ABC television. September 28, 2000

7 Lowry RT. An end-of-shift tale. Ann Emerg Med 1998;31(02):287-288

8 Young RS. Young M.D.'s long hours ill-advised? Sleep deprivation leads to horror tales. Rochester Democrat and Chronicle. October 21,1990

9 Wendt JR, Yen LJ. The resident by moonlight: a misguided missile. JAMA 1988;259(01):43-44

10 Worth R. Exhaustion that kills: why residents are still overworkedand what we can do about it. Wash Mon 1999;3(01):15-20

11 Steele MT, Ma OJ, Watson WA, Thomas HA Jr, Muelleman RL. The occupational risk of motor vehicle collisions for emergency medicine residents. Acad Emerg Med 1999;6(10): 1050-1053

12 Friedman RC, Kornfeld DS, Bigger TJ. Psychological problems associated with sleep deprivation in interns. J Med Educ 1973;48(05): 436-441

13 Leonard C, Fanning N, Attwood J, Buckley M. The effect of fatigue, sleep deprivation and onerous working hours on the physical and mental wellbeing of pre-registration house officers. Ir J Med Sci 1998;167(01):22-25

14 Lingenfelser T, Kaschel R, Weber A, Zaiser-Kaschel H, Jakober B, Küper J. Young hospital doctors after night duty: their taskspecific cognitive status and emotional condition. Med Educ 1994;28(06):566-572

15 Hart RP, Buchsbaum DG, Wade JB, Hamer RM, Kwentus JA. Effect of sleep deprivation on first-year residents' response times, memory, and mood. J Med Educ 1987;62(11):940-942

16 Friedman RC, Bigger JT, Kornfeld DS. The intern and sleep loss. N Engl J Med 1971;285(04):201-203

17 Samkoff JS, Jacques $\mathrm{CH}$. A review of studies concerning effects of sleep deprivation and fatigue on residents' performance. Acad Med 1991;66(11):687-693

18 Valko RJ, Clayton PJ. Depression in the internship. Dis Nerv Syst 1975;36(01):26-29

19 Pitts FN Jr, Schuller AB, Rich CL, Pitts AF. Suicide among U.S. women physicians, 1967-1972. Am J Psychiatry 1979;136(05):694-696

20 Reuben DB. Depressive symptoms in medical house officers. Effects of level of training and work rotation. Arch Intern Med 1985;145(02):286-288

21 Reuben DB. Psychologic effects of residency. South Med J 1983;76 (03):380-383

22 Mozurkewich EL, Luke B, Avni M, Wolf FM. Working conditions and adverse pregnancy outcome: a meta-analysis. Obstet Gynecol 2000;95(04):623-635

23 Osborn LM, Harris DL, Reading JC, Prather MB. Outcome of pregnancies experienced during residency. J Fam Pract 1990;31 (06):618-622

24 Klebanoff MA, Shiono PH, Rhoads GG. Outcomes of pregnancy in a national sample of resident physicians. N Engl J Med 1990;323 (15):1040-1045
25 Klebanoff MA, Shiono PH, Rhoads GG. Spontaneous and induced abortion among resident physicians. JAMA 1991;265(21): 2821-2825

26 Glicksman E. Wanting It All: A New Generation of Doctors Places Higher Value on Work-Life Balance. AAMC Reporter. https://www. aamc.org/newsroom/reporter/336402/work-life.html. Accessed on November 25, 2018

27 IHS Inc. Association of American Medical Colleges. The Complexities of Physician Supply and Demand: Projections from 2013 to 2025. Washington, DC: Association of American Medical Colleges; 2015

28 Wanous JP, Poland TD, Premack SL, Davis KS. The effects of met expectations on newcomer attitudes and behaviors: a review and meta-analysis. J Appl Psychol 1992;77(03):288-297

29 Gregory Irving P, Montes SD. Met expectations: the effects of expected and delivered inducements on employee satisfaction. J Occup Organ Psychol 2009;82(02):431-451

30 Schwab RL, Jackson SE, Schuler RS. Educator burnout: sources and consequences. Educ Res Q 1986;10(03):14-30

31 Locke EA, Latham GP. Building a practically useful theory of goal setting and task motivation. A 35-year odyssey. Am Psychol 2002; 57(09):705-717

32 Locke E, Latham G. New Developments in Goal Setting and Task Performance. New York, NY: Routledge; 2013

33 Miner JB. The rated importance, scientific validity, and practical usefulness of organizational behavior theories: a quantitative review. Acad Manag Learn Educ 2003;2(03):250-268

34 Lee P, Earley C. Comparative peer evaluations of organizational behavior theories. Organ Dev J 1992;10(04):37-42

35 Pinder CC. Work Motivation: Theory, Issues, and Applications. Upper-Saddle River, NJ: Prentice Hall; 1998

36 Barron KE, Harackiewicz JM. Achievement goals and optimal motivation: testing multiple goal models. J Pers Soc Psychol 2001;80(05):706-722

37 Schroen AT, Brownstein MR, Sheldon GF. Comparison of private versus academic practice for general surgeons: a guide for medical students and residents. J Am Coll Surg 2003;197(06): $1000-1011$

38 DeCastro R, Griffith KA, Ubel PA, Stewart A, Jagsi R. Mentoring and the career satisfaction of male and female academic medical faculty. Acad Med 2014;89(02):301-311

39 Sullivan MC, Bucholz EM, Yeo H, Roman SA, Jr RHB, Sosa JA. Join the Club. 2012;147(05):408-414

40 Ahmadiyeh N, Cho NL, Kellogg KC, et al. Career satisfaction of women in surgery: perceptions, factors, and strategies. J Am Coll Surg 2010;210(01):23-28

41 Troppmann KM, Palis BE, Goodnight JE Jr, Ho HS, Troppmann C. Women surgeons in the new millennium. Arch Surg 2009;144 (07):635-642

42 Streu R, McGrath MH, Gay A, Salem B, Abrahamse P, Alderman AK. Plastic surgeons' satisfaction with work-life balance: results from a national survey. Plast Reconstr Surg 2011;127(04): $1713-1719$

43 U.S. Department of Labor. Bureau of Labor Statistics. Occupational Outlook Handbook. https://www.bls.gov/ooh/about/ooh-faqs. htm. Accessed April 4, 2018

44 Coleman J, Coleman J. How Two-Career Couples Stay Happy. Harvard Business Review. https://hbr.org/2012/07/how-twocareer-couples-stay-ha. Accessed April 4, 2018

45 Wittenberg-Cox A. Being a Two-Career Couple Requires a LongTerm Plan. Harvard Business Review. https://hbr.org/2018/02/ being-a-two-career-couple-requires-a-long-term-plan. Accessed April 4, 2018

46 Schulte B. Time in the bank: a Stanford plan to save doctors from burnout. The Washington Post. August 20, 2015

47 Grimm LJ, Nagler A, Maxfield CM. Survey of the incidence and effect of major life events on graduate medical education trainees. Med Educ Online 2015;20(01):27597 
48 Sullivan MC, Yeo H, Roman SA, Bell RH Jr, Sosa JA. Striving for work-life balance: effect of marriage and children on the experience of 4402 US general surgery residents. Ann Surg 2013;257 (03):571-576
49 Sandler BJ, Tackett JJ, Longo WE, Yoo PS. Pregnancy and parenthood among surgery residents: results of the first nationwide survey of general surgery residency program directors. J Am Coll Surg 2016;222(06):1090-1096 